\title{
New Design Compounds for Bone Cancer Treatment: Broader Bioactivity of Silicon Modified Methotrexate
}

\author{
Esma Eryılmaz Doğan* \\ ${ }^{1}$ Department of Biomedical Engineering, Faculty of Technology, Selcuk University, Turkey, (ORCID ID 0000-0001-6809-7513), *(eeryilmaz@selcuk.edu.tr)
}

(1st International Conference on Applied Engineering and Natural Sciences ICAENS 2021, November 1-3, 2021)

(DOI: 10.31590/ejosat.1019547)

ATIF/REFERENCE: Eryılmaz Doğan, Esma (2021). New Design Compounds for Bone Cancer Treatment: Broader Bioactivity of Silicon Modified Methotrexate. European Journal of Science and Technology, (28), Xx-Xx.

\begin{abstract}
Complex diseases such as cancer are mostly described by combining negative effects of multiple biological factors or pathways. Based on that, multi-targeted approach for treating cancer is gaining interest. The aim of this study is to introduce a computational approach and to design new, multi-targeted drug candidates for treatment of bone cancer. In this approach, the FDA approved drugs of bone cancer were evaluated in terms of their molecular pharmaceutical properties and their bioactivity parameters predicted by bioinformatics and cheminformatics softwares. Among them, Methotrexate was chosen as a lead molecule due to its broader spectrum of bioactivity on the most important drug targets reported in literature. The lead molecule was exposed to basic bioisosteric modifications to obtain a better drug compound with improved bioactivity and a stronger drug-likeness profile using the known drug structure. Design compounds produced by a number of bioisosteric modifications performed on the $2 \mathrm{D}$ structure of the lead compound were evaluated in terms of both criteria; bioactivity and drug-likeness. Silicone modified compounds M4, M13, M14, and M15 showed a much broader spectrum of biological activity than that of the approved compound Methotrexate. The interesting effect of silicone incorporation makes our compounds promising drug candidates for further pharmaceutical investigation.
\end{abstract}

Keywords: in silico, computer aided drug design, bone cancer, ADME, bioactivity, drug-likeness 


\section{Introduction}

Bioinformatics have been found a common place among biomedical sciences along with the exponentially increasing number of technological devices used in the experimental bioscience. It is extensively used in the field of drug design and discovery to decrease the laboratory cost and to help with understanding the experimental observations. So called with Computer Aided Drug Design (CADD) not only fastens the actual drug discovery process, but also diminishes the actual cost required to perform the whole new drug development steps. Since the CADD is basically dealing with a number of molecular database, molecular physico-chemical, pharmacological, and structural properties, bioinformatics is the central field of drug development $[4,5]$. During the development process, possible therapeutic agents can be modeled using molecular modeling softwares, and their molecular structural properties can be calculated using quantum chemical calculations. Using molecular editing softwares, a variety of possible modifications are created, and their molecular properties are recalculated to improve desired combinations of physico-chemical properties and to decrease possible side effects of the new design drug candidates for a disease of interest.

Molecular editing or designing new molecules are important part of the early stage of CADD. For that, bioisosterism is commonly used among researchers to design new therapeutic agents using the known drug structures. Bioisosteres are defined by Burger as 'Compounds of groups that possess near-equal molecular shapes and volumes, approximately the same distribution of electrons, and which exhibit similar physical properties' [7]. Using the definition and the members of bioisosteric groups reported in literature and summarized partially in Table 1, we can create different design compounds, calculate their physico-chemical properties using bioinformatics and cheminformatics softwares, and evaluate them in terms of druglikeness criteria. The most promising candidates with a higher drug-likeness score and with a broader bioactivity profile are chosen for further pharmacological research such as synthesizing the compounds, examining 'in vitro' cell interaction parameters, animal testing, and clinical trials, respectively.

Bone and joint cancer (sarcomas) is rare, but it is mostly seen in children and young adults. It can be malignant and can destroy nearby tissue and distant organs. According to the National Institute of Health (NIH), an estimated 3600 people from the U.S population will be diagnosed this year and approximate $11 \%$ of these cases will occur at the age of 15 to 19 . The estimated death is reported as 1720 people in 2020 (https://www.cancer.gov/). Based on the cases reported in between 2010-2016, the 5-year survival rate is given as $66 \%$ which need to be improved. And yet, current drugs used in the treatment of bone cancer causes side effects including hearing, fertility, and neurological problems. On the basis of the statistics, better alternatives to the current approved bone cancer drugs with improved pharmacological effect is crucial.

For complex diseases such as cancer, multiple factors or pathways contribute to the disease process simultaneously $[9,10]$. Based on the fact, cancer treatment agents are recently designed to target multiple proteins and/or to interact with multiple pathways. So called multi-targeted therapy has growing interest in last a few decades $[11,12]$. We, therefore, discuss, in this study, 'in silico' drug design with multi-targeted manner meaning a broad range of biological activity on the most important drug targets to develop new therapeutic agents for the treatment of bone cancer.

\section{Material and Method}

The FDA (The U.S Food and Drug Administration) approved small molecule drugs used for bone cancer treatment were extracted from the official website of the National Cancer Institute, the National Institute of Health of USA (NIH), https://www.cancer.gov/. SMILES codes taken from the unique bioinformatics and cheminformatics resource DrugBank 5.1.1 https://www.drugbank.ca/ [13] were used to perform all the analysis.

\subsection{Design Compound Preparation}

Different types of information about cancer including the approved drugs for treatment, cancer prevention, diagnosis, or clinical trials can be found in the central website of https://www.cancer.gov, supported by the National Cancer Institute of U.S. After listing the approved drugs for bone cancer treatment, the detailed information including SMILES codes, 2D structures, and 3D structures were taken from the DrugBank database. Using the SMILES, editing and/or modifying the 2D structure of the marketed drugs, the novel structures were created by Molinspiration software. The new design structures were then investigated in terms of their drug-likeness score and bioactivity properties.

\subsection{Evaluation of Drug-Likeness}

Investigation of the approved drugs showed that some basic molecular properties can be used to evaluate a compound, if it was a good oral drug candidate or not. These properties are composed of some structural and physico-chemical properties of a molecule such as lipophilicity, polar surface area, the number of hydrogen bond donor and the number of hydrogen bond acceptor. Based on this fact, different groups were listed in literature with their druglikeness criteria using the basic molecular properties. In this study, five different drug likeness criteria reported by Lipinski [1], Ghose [2], Veber [6], Egan [8], and Muegge [3] were used to evaluate the approved drugs and new design molecules.

\subsection{Prediction of Bioactivity}

Drug molecules and new design compounds were evaluated according to their biological activity across the most targeted class of molecules such as kinase inhibitors, G-protein coupled receptors (GPCR), nuclear receptors, ion channel modulators, protease, and enzyme inhibitors by using Molinspiration software. Online cheminformatics tool SwissADME, http://swissadme.ch/, was used for the detailed search of drug likeness properties and ADME parameters.

\subsection{Bioisosterism}

Bioisosters are defined as atoms or group of atoms which have different structure but are recognized similarly by biosystems. Therefore, we used the concept of bioisosterism to create new bioactive compounds against bone cancer using the known structure of the approved drugs. Classical bioisosters grouped as shown in Table 1. 
Table 1. The classical bioisosteric groups

\begin{tabular}{ll}
\hline Classes & Bioisosteric groups \\
\hline Monovalent & $-\mathrm{OH}, \mathrm{NH}_{2},-\mathrm{CH}_{3},-\mathrm{F},-\mathrm{Cl},-\mathrm{Br},-\mathrm{Si}_{3}$ \\
Divalent & $\mathrm{C}=\mathrm{C}, \mathrm{C}=\mathrm{N}, \mathrm{C}=\mathrm{O}, \mathrm{C}=\mathrm{S},-\mathrm{CH}_{2}--\mathrm{NH}-,-\mathrm{O}-,-\mathrm{S}-$ \\
Trivalent & $-\mathrm{CH}=,-\mathrm{N}=$ \\
Tetravalent &
\end{tabular}

\section{Results and Discussion}

We investigated the approved small molecule drugs used in the treatment of bone cancer obtained from the central cancer website of the U.S., https://www.cancer.gov. That was concluded with three molecules listed in Table 2 . We examined both of the approved drugs listed in the Table 2 and the new design compounds created by bioisosteric modifications in terms of two classifications; drug-likeness score and biological activity across the most important drug targets.

\subsection{Physico-chemical Properties and Drug- likeness}

Drug-likeness score is evaluated based on the combination of the physico-chemical and the structural properties of the molecule $[14,15]$. Based on these combinations, drug-likeness criteria are reported in literature by different group of researchers. Small molecule drug candidates are evaluated in terms of those molecular properties such as $\log \mathrm{P}(\mathrm{P}$ is the calculated partition coefficient), molecular weight, number of rotatable bonds, number of hydrogen bond donor and hydrogen bond acceptor to evaluate to what level the molecule close to be a good bioavailable drug. $\log \mathrm{P}$ value is often used in drug discovery process to describe the molecular hydrophobicity or lipophilicity. Negative Log P value represents hydrophilic nature, while a positive Log P value indicates hydrophobicity. It is also used in many pharmacology related terms such as toxicity, solubility (Log S), even binding affinity of the compound to a target molecule.

Table 2. The names, the 2D structures and the physico-chemical properties of the marketed drugs used for bone cancer treatment were listed. ${ }^{a}$ Logarithm of partition coefficient (MiLog P), ${ }^{b}$ Molecular weight in the unit of gr/mol (MW), ${ }^{\mathrm{c}}$ Topological polar surface area

(TPSA) in the unit of $\AA$, ${ }^{\mathrm{d}}$ The number of hydrogen bond donor and eacceptor.

\begin{tabular}{|c|c|c|c|c|c|c|}
\hline Drug Name & 2D structure & MiLogPa $^{a}$ & $\mathbf{M W}^{\mathbf{b}}$ & TPSA $^{c}$ & $\mathrm{nOH}^{\mathrm{d}}$ & $\mathrm{nOHNH}^{\mathrm{e}}$ \\
\hline & & 0.78 & 1255.4 & 359.9 & 28 & 6 \\
\hline
\end{tabular}

Methotrexate 
Molecular properties of the approved drugs Dactinomycin, Doxorubicin, and Methotrexate were summarized in Table 2 and drug-likeness criteria were provided in the second column Table 3. From our list, Dactinomycin and Doxorubicin showed a low hydrophobic nature with the score of 0.78 and 0.57 , respectively, while Methotrexate resulted in a hydrophilic nature with the score of -1.97 , which were all fall in the range of the criteria to be less than 5. Topological polar surface area, on the other hand, is another criterion to be confirmed as less than $150 \AA^{2}$ according to Muegge's criteria shown in Table 3. Our calculations showed that all of three molecules violated that which was interpreted as possible reasons of the negative side effects of the drug compounds to take care during the process of designing new molecules. Possible modifications should be in a way of decreasing polarity of the molecular surface to create better design drug compounds.

The numbers of rotatable bonds are also restricted to have a more stable molecule and a less number of possible conformations to be tested in the experimental process. Molecular weight should also be equal or less than 500 due to the ease solubility and the diffusion of the molecule trough lipid bilayer membrane. More explanation regarding the rest of the molecular pharmaceutical descriptors can be found in our previous study [16]. From our list of drug molecules, we have seen that all molecules except Methotrexate violated the criteria with a quite high molecular weight. Methotrexate obeys the rule with a weight of 454.4 .

According to the mostly known Lipinski's criteria called 'rule of five', molecular weight should be less than 500, miLog P value less than 5, the number of hydrogen bond donor and hydrogen bond acceptor should be less than 5 and 10, respectively [1] Overall, when we evaluate the three drugs approved for bone cancer, Dactinomycin and Doxorubicin showed quite negative profile in terms of physico-chemical properties and drug-likeness profile as shown in Table 3. Specifically, Dactinomycin violated all of the criteria with excess molecular weight and higher number of hydrogen bond donor. Methotrexate, on the other hand, remained a more promising structure as a good bioavailable lead molecule structure. Therefore, for our further investigation, the structure of Methotrexate was used as a lead structure.

Table 3. Drug-likeness criteria reported in the literature. MW: Molecular weight. nOHNH: Number of hydrogen bond donors. nOH: Number of hydrogen bond acceptors. mLog P: Logarithm of partition coefficient. TPSA: Topological polar surface area. MR: Molar refractivity. Nat: Number of heavy atoms. Nrotb: Number of rotatable bonds. Nrings: Number of rings. Ncarbon: Number of carb on atoms.

\section{Drug-likeness criteria}

Dactinomycin Doxorubicin $\underline{\text { Methotrexate }}$

\begin{tabular}{lllll}
\hline Lipinski 2001[1] & $\mathrm{MW} \leq 500, \mathrm{nOH} \leq 10$ & No, 2 vio. & No, 3 vio.
\end{tabular}

$\mathrm{mLog} \mathrm{P} \leq 4.15, \mathrm{nOHNH} \leq 5$

\begin{tabular}{llllll}
\hline Ghose $1999[2]$ & $160 \leq \mathrm{MW} \leq 480$, & $-0.4 \leq \mathrm{W} \log \mathrm{P} \leq 5.6$ & No, 4 vio. & No, 2 vio. & Yes
\end{tabular}

$40 \leq \mathrm{MR} \leq 130, \quad 20 \leq$ atoms $\leq 70$

\begin{tabular}{lllll}
\hline Muegge $2001[3]$ & $200 \leq \mathrm{MW} \leq 600$, & $-2 \leq \mathrm{xLogP} \leq 5$ & No, 3 vio. & No, 3 vio.
\end{tabular}

TPSA $\leq 150$, Nrotb $\leq 15$, Nrings $\leq 7$, Ncarbon $>4$

$\mathrm{nOHNH} \leq 5, \mathrm{nOH} \leq 10$

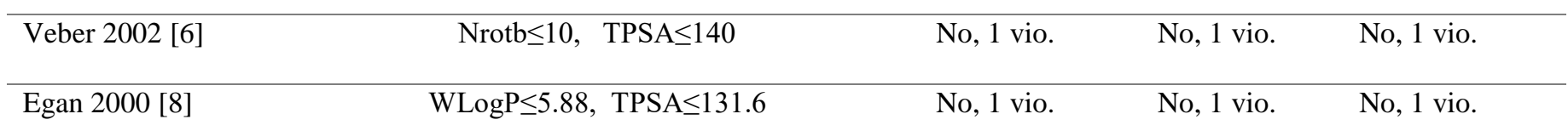

\subsection{Evaluation of Multi-Targeted Bioactivity}

The second approach we applied to the compounds is the evaluation of bioactivity scores. Computational bioactivity score is basically an indicator of how strong the molecule interacts with the target molecule which is mostly a protein. The virtual screening results of the Molinspiration software are represented as color coded. Positive activity scores equal or higher than 0.50 
texts in dark green indicating a good bioactivity behavior and the scores between 0.20 and 0.5 is considered to represent a moderate bioactivity by coloring with light green.

For designing process, we first calculated the activity scores for the approved drugs and the results were shown in Table 4. Dactinomycin showed no bioactivity with all negative scores on the target proteins. Doxorubicin and Methotrexate, on the other hand, showed bioactivity on certain targets. While Doxorubicin was resulted in a good activity with a score of 0.67 and 0.66 across protease and enzyme inhibitors, respectively, Methotrexate showed a good activity across GPCR and enzyme inhibitors with the scores of 0.51 and 0.72 and a moderate activity on ion channel moderator, kinase, and protease inhibitors with the scores of $0.23,0.38$, and 0.27 , respectively. GPCR, among other targets, was reported as the most targeted class of compounds [17]. Therefore, Methotrexate was chosen as a lead molecule for further investigation due to both its better drug-likeness profile and its higher bioactivity score.
Table 4: Biological activity scores of the approved drugs for bone cancer across G-Protein CR, ion channel modulator (ICM), kinase inhibitors (KI), nuclear receptor ligands (NRL), and protease $(\mathrm{PI})$ and enzyme inhibitors $(\mathrm{EI})$.

\begin{tabular}{l|llllll}
\hline Drug Name & GPCR & $I C M$ & $K I$ & NRL & $P I$ & $E I$ \\
\hline Dactinomycin & -0.38 & -3.95 & -3.96 & -3.96 & -3.83 & 3.90 \\
Doxorubicin & 0.20 & -0.20 & -0.07 & -0.32 & 0.67 & 0.66 \\
Methotrexate & 0.51 & 0.23 & 0.38 & -0.38 & 0.27 & 0.72 \\
& & & & & & \\
\hline
\end{tabular}

\subsection{Physico-chemical Properties}

For obtaining new design multi-targeted therapeutic agents, we tried to improve the drug-likeness profile and the number of activity shown across the drug targets. In order to obtain a better alternative than the marketed drug Methotrexate, the molecular structure of Methotrexate shown in Figure 1 was exposed to modifications using monovalent bioisosteres listed in Table 1. The modifications were replaced to the end of $\mathrm{X}$ and $\mathrm{Y}$ shown in the Figure 1. The new design structures were re-evaluated in terms of bioactivity and the results were tabulated. The process was repeated till obtaining a broader spectrum of activity than that of the approved drug Methotrexate. The fifteen analogs of Methotrexate listed in Table 5 were investigated to obtain a better structure than Methotrexate itself. Our investigation was resulted in that silicon based structures such as M4, M13, M14, and M15 interestingly possessed an improved bioactivity on almost all of the drug targets. We also re-evaluated these promising compounds in terms of their physico-chemical properties and drug-likeness criteria to decrease the number of possible structure for testing in experimental process and decreasing the laboratory cost. Their drug-likeness profiles were similar to that of Methotrexate, so we believed that these new design compounds were worth to try in the next steps of drug development.

Table 5. Bioactivity scores of Methotrexate and its fifteen analogs across the most important drug targets predicted by Molinspiration. GPCR G-Protein coupled receptor, ICM ion channel modulator, KI kinase inhibitors, NRL nuclear receptor ligands, and PI protease inhibitors and EI enzyme inhibitors.

\begin{tabular}{lllllllll}
\hline Compound & $\mathbf{X}$ & $\mathbf{Y}$ & GPCR & ICM & KI & NRL & PI & EI \\
\hline Methotrexate & $\mathrm{COOH}$ & $\mathrm{CH}_{3}$ & 0.51 & 0.23 & 0.38 & -0.38 & 0.27 & 0.72 \\
& & & & & & & & \\
\hline M1 & $\mathrm{B}(\mathrm{OH})_{2}$ & $\mathrm{CH}_{3}$ & 0.46 & 0.22 & 0.42 & -0.29 & 0.65 & 1.20 \\
\hline M2 & $\mathrm{NH}_{2}$ & $\mathrm{CH}_{3}$ & 0.62 & 0.35 & 0.53 & -0.44 & 0.39 & 0.80 \\
\hline M3 & $\mathrm{SiH}_{3}$ & $\mathrm{CH}_{3}$ & 0.55 & 0.29 & 0.44 & -0.43 & 0.29 & 0.78 \\
\hline M4 & $\mathrm{Si}\left(\mathrm{CH}_{3}\right)_{3}$ & $\mathrm{CH}_{3}$ & 0.76 & 0.49 & 0.60 & -0.40 & 0.53 & 1.07 \\
\hline M5 & $\mathrm{COOH}$ & $\mathrm{SiH}_{3}$ & 0.47 & 0.16 & 0.34 & -0.47 & 0.27 & 0.63 \\
& & & & & & & & \\
\hline M6 & $\mathrm{COOH}$ & $\mathrm{Si}\left(\mathrm{CH}_{3}\right)_{3}$ & 0.54 & 0.22 & 0.39 & -0.43 & 0.38 & 0.73
\end{tabular}




\begin{tabular}{lllllllll}
\hline M7 & $\mathrm{COOH}$ & $\mathrm{OH}$ & 0.30 & 0.04 & 0.16 & -0.66 & 0.29 & 0.64 \\
& & & & & & & & \\
\hline M8 & $\mathrm{COOH}$ & $\mathrm{F}$ & 0.47 & 0.16 & 0.35 & -0.46 & 0.27 & 0.63 \\
& & & & & & & & \\
\hline M9 & $\mathrm{COOH}$ & $\mathrm{Cl}$ & 0.47 & 0.16 & 0.34 & -0.47 & 0.26 & 0.63 \\
& & & & & & & & \\
\hline M10 & $\mathrm{BH}_{2}$ & $\mathrm{OH}$ & 0.26 & -0.06 & 0.17 & -0.73 & 0.30 & 0.75 \\
& & & & & & & & \\
\hline M11 & $\mathrm{B}(\mathrm{OH})_{2}$ & $\mathrm{OH}$ & 0.25 & -0.05 & 0.20 & -0.57 & 0.67 & 1.12 \\
& & & & & & & & \\
\hline M13 & $\mathrm{Si}\left(\mathrm{CH}_{3}\right)_{3}$ & $\mathrm{OH}$ & 0.55 & 0.23 & 0.39 & -0.67 & 0.55 & 0.99 \\
& & & & & & & & \\
\hline M14 & $\mathrm{Si}\left(\mathrm{CH}_{3}\right)_{3}$ & $\mathrm{~B}(\mathrm{OH})_{2}$ & 0.67 & 0.37 & 0.57 & -0.35 & 0.67 & 1.08 \\
& & & & & & & & \\
\hline M15 & $\mathrm{Si}\left(\mathrm{CH}_{3}\right)_{3}$ & $\mathrm{~F}$ & 0.71 & 0.42 & 0.57 & -0.48 & 0.52 & 0.98 \\
& & & & & & & & \\
\hline
\end{tabular}

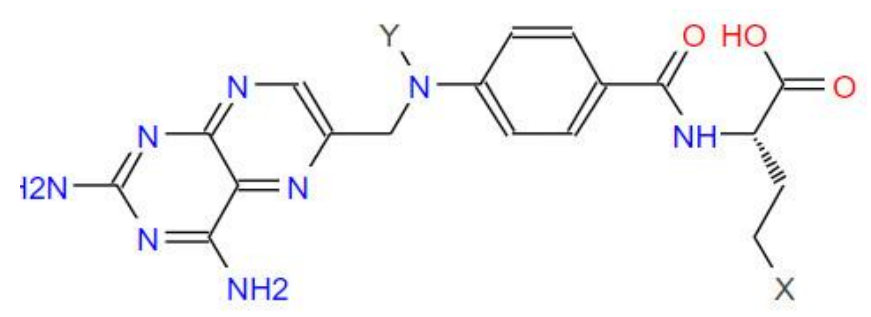

Fig. 1. 2D molecular structure of Methotrexate with $\mathrm{X}$ and $\mathrm{Y}$ substitutions to be used in Table 5 .

\section{Conclusion}

In our in silico drug design approach, we first investigated the existing FDA approved bone cancer drugs in terms of their computationally predicted physico-chemical properties, different drug-likeness criteria, and their biological activity across the important drug targets GPCR, in channel modulator, kinase inhibitors, nuclear receptor ligands, protease, and enzyme inhibitors. The drug molecule Methotrexate showing a higher number of bioactivity was considered as that drug structure could be used as a lead molecule for further investigation and it was taken into the process of monovalent bioisosteric replacements. From the series of bioisosteric analogues of the marketed drug Methotrexate, the compounds M4, M13, M14, and M15 showed a much broader spectrum of bioactivity. Especially M4 showed a good activity on the five drug targets over the six tested. Its druglikeness properties were also checked and were found similar to the marketed drug Methotrexate. Therefore, our in silico design compounds M4, M13, M14, and M15 give a promising insight with a multi-targeted manner for further pharmacological research.

\section{References}

1. Lipinski, C.A., (2001). Avoiding investment in doomed drugs. Curr Drug Discov, 1: p. 17-19.

2. Ghose, A.K., V.N. Viswanadhan, and J.J. Wendoloski, (1999). A knowledge-based approach in designing e-ISSN: 2148-2683 combinatorial or medicinal chemistry libraries for drug discovery. 1. A qualitative and quantitative characterization of known drug databases. Journal of combinatorial chemistry, 1(1): p. 55-68.

3. Muegge, I., S.L. Heald, and D. Brittelli, (2001). Simple selection criteria for drug-like chemical matter. Journal of Medicinal Chemistry, 44(12): p. 1841-1846.

4. Schmidt, T., A. Bergner, and T. Schwede, (2014). Modelling three-dimensional protein structures for applications in drug design. Drug discovery today, 19(7): p. 890-897.

5. Geldenhuys, W.J., et al., (2006). Optimizing the use of opensource software applications in drug discovery. Drug Discovery Today. 11(3-4): p. 127-132.

6. Veber, D.F., et al., (2006). Molecular properties that influence the oral bioavailability of drug candidates. Journal of medicinal chemistry. 45(12): p. 2615-2623.

7. Burger, A., (1991). Isosterism and bioisosterism in drug design, in Progress in Drug Research/Fortschritte der Arzneimittelforschung/Progrès des recherches pharmaceutiques. Springer. p. 287-371.

8. Egan, W.J., K.M. Merz, and J.J. Baldwin, (2000). Prediction of drug absorption using multivariate statistics. Journal of Medicinal Chemistry. 43(21): p. 3867-3877.

9. Zheng, H., M. Fridkin, and M. Youdim, (2014). From single target to multitarget/network therapeutics in Alzheimer's therapy. Pharmaceuticals (Basel). 7(2): p. 113-35.

10. Petrelli, A. and S. Giordano, (2008). From single- to multitarget drugs in cancer therapy: when aspecificity becomes an advantage. Curr Med Chem. 15(5): p. 422-32.

11. Li, Y., et al., (2014). Multi-targeted therapy of cancer by niclosamide: A new application for an old drug. Cancer letters. 349(1): p. 8-14.

12. Berquin, I.M., I.J. Edwards, and Y.Q. Chen, (2008). Multitargeted therapy of cancer by omega-3 fatty acids. Cancer letters. 269(2): p. 363-377.

13. Wishart, D.S., et al., (2018). DrugBank 5.0: a major update to the DrugBank database for 2018. Nucleic Acids Res. 46(D1): p. D1074-D1082.

14. Dogan, E. E. (2021). Computational Bioactivity Analysis and Bioisosteric Investigation of the Approved Breast Cancer 
Drugs Proposed New Design Drug Compounds: Increased Bioactivity Coming with Silicon and Boron. Letters in Drug Design \& Discovery, 18, 1-11.

15. Dogan, E. E. (2021). Computational binding analysis and toxicity evaluation of estrogen receptor with estradiol and the approved SERMs raloxifene, tamoxifen, and toremifene. Medicine, 10, 157-61.

16. Eryilmaz, E., (2019). Multi-targeted anti-leukemic drug design with the incorporation of silicon into Nelarabine: How silicon increases bioactivity. European Journal of Pharmaceutical Sciences. 134: p. 266-273.

17. Eder, J., R. Sedrani, and C. Wiesmann, (2014). The discovery of first-in-class drugs: origins and evolution. Nature Reviews Drug Discovery.. 13(8): p. 577. 